\title{
Publisher Correction: Molnupiravir: coding for catastrophe
}

Brandon Malone and Elizabeth A. Campbell

Correction to: Nature Structural \& Molecular Biology https://doi.org/10.1038/s41594-021-00657-8, published online 13 September 2021. In the version of this News \& Views initially published, there were errors in the main text and in Fig. 1

Specifically, in the second paragraph, there was a typo in the trade name for remdesivir (Veklury), which appeared as "Verklury." In the fourth paragraph, fifth sentence, beginning "After incorporation of molnupiravir," the end of the sentence has been modified to read "but is readily incorporated into the p-RNA" rather than the original "nascent RNA." In the fifth paragraph, "Gordon et al." replaces "Gorden et al.'." In Fig. 1f, the boxed area to the right under the "Mutagenesis" label, the base pair now reading "M $\rightarrow$ A" originally appeared as " $\mathrm{M} \rightarrow \mathrm{G}$."

The online version of the article has been updated.

Published online: 11 October 2021

https://doi.org/10.1038/s41594-021-00683-6

(c) Springer Nature America, Inc. 2021 